\title{
Aplicabilidad de métodos de evaluación de la amenaza sísmica para un proyecto minero (basada en datos neotectónicos, instrumentales e históricos), Andes Centrales, San Juan, Argentina
}

\author{
Luciano Oldecop ${ }^{1, *}$, Laura Perucca ${ }^{2,3}$ \\ ${ }^{1}$ Instituto de Investigaciones Antisísmicas “Ing. Aldo Bruschi”, Universidad Nacional de San Juan. Av. Libertador General San \\ Martín, 5400 San Juan, Argentina. \\ ${ }^{2}$ CONICET (Consejo de Investigaciones Científicas y Técnicas). \\ ${ }^{3}$ Gabinete de Neotectónica, Instituto de Geología Dr. Emiliano Aparicio - Facultad de Ciencias Exactas, Físicas y \\ Naturales, Universidad Nacional de San Juan. Av. Ignacio de la Roza y Meglioli 1290. 5400 San Juan, Argentina. \\ *oldecop@unsj.edu.ar
}

\section{Resumen}

En el presente trabajo se presenta la metodología aplicada en el análisis de la amenaza sísmica para un sitio específico de los Andes argentinos. Se recopiló y analizó la información neotectónica y sismológica como tarea inicial del trabajo. Como es habitual en muchas regiones de Sudamérica, la información sismológica y paleosismológica disponible es escasa y, en general, insuficiente para definir acabadamente la actividad sísmica en el espacio y en el tiempo. Los criterios de verificación sísmica de las obras involucradas en el proyecto se definieron sobre la base de la práctica internacional. Las metodologías determinística y probabilística se aplicaron para la evaluación de la amenaza sísmica en el sitio del proyecto. Los resultados obtenidos se comparan en este trabajo y se discuten los procedimientos a utilizar para la selección de los movimientos sísmicos de diseño. Finalmente se define un criterio de trabajo y se presentan los movimientos sísmicos de diseño que resultaron del estudio.

Palabras clave: amenaza sísmica, neotectónica, métodos determinísticos y probabilísticos.

\begin{abstract}
In the present paper the methodology applied for the evaluation of the seismic hazard in a specific site in the Argentinean Andes is presented. Available neotectonic and seismological information was compiled and analyzed as a preliminary work. As it is usual in most South American regions, background seismological and paleosismological information is scarce and, in general, insufficient to completely define the seismic activity in space and time. The definition of seismic design criteria for all the works involved in the project was done with basis on international practice. Both, deterministic and probabilistic analysis methodologies were applied to evaluate the seismic threat in the project's site. The obtained results are compared in this work and the available procedures for selecting the seismic design motions are discussed. Finally, a working criterion is defined and the resulting seismic design motions obtained from the study are presented.
\end{abstract}

Keywords: seismic hazard, neotectonics, deterministic and probabilistic methods. 


\section{Introducción}

El proyecto minero Casposo se ubica al pie de la Cordillera Frontal, a 2400 m.s.n.m. y a 24 km al NE del poblado Calingasta, en la provincia de San Juan, centrooeste argentino (Figura 1). Se trata de la explotación a cielo abierto y subterránea de un depósito metalífero (oro y plata). Los residuos generados consisten en escombros y las colas del proceso de extracción metalífera, las que serán sometidas a un proceso de filtrado con el fin de extraer una parte del agua de proceso para su reutilización. Las principales obras a ejecutar en el proyecto son: mina a cielo abierto, mina subterránea, planta de procesamiento, escombrera, pila de colas y edificaciones auxiliares. El diseño y análisis de seguridad requiere de la definición de las solicitaciones sísmicas que se esperan en el sitio de emplazamiento del proyecto.

La caracterización sísmica de una región involucra la evaluación del potencial sísmico de las fallas o estructuras tectónicas singulares, desde el punto de vista de la aplicación al cálculo de la amenaza sísmica. Concretamente, interesan aquellas fallas que presenten evidencias de actividad durante el Cuaternario, o claras asociaciones con la actividad sísmica. Para incorporar de modo efectivo una falla en el cálculo de la amenaza sísmica es necesario estimar, en primer lugar, el terremoto máximo (o magnitud máxima) que la falla es capaz de producir, basándose en las dimensiones
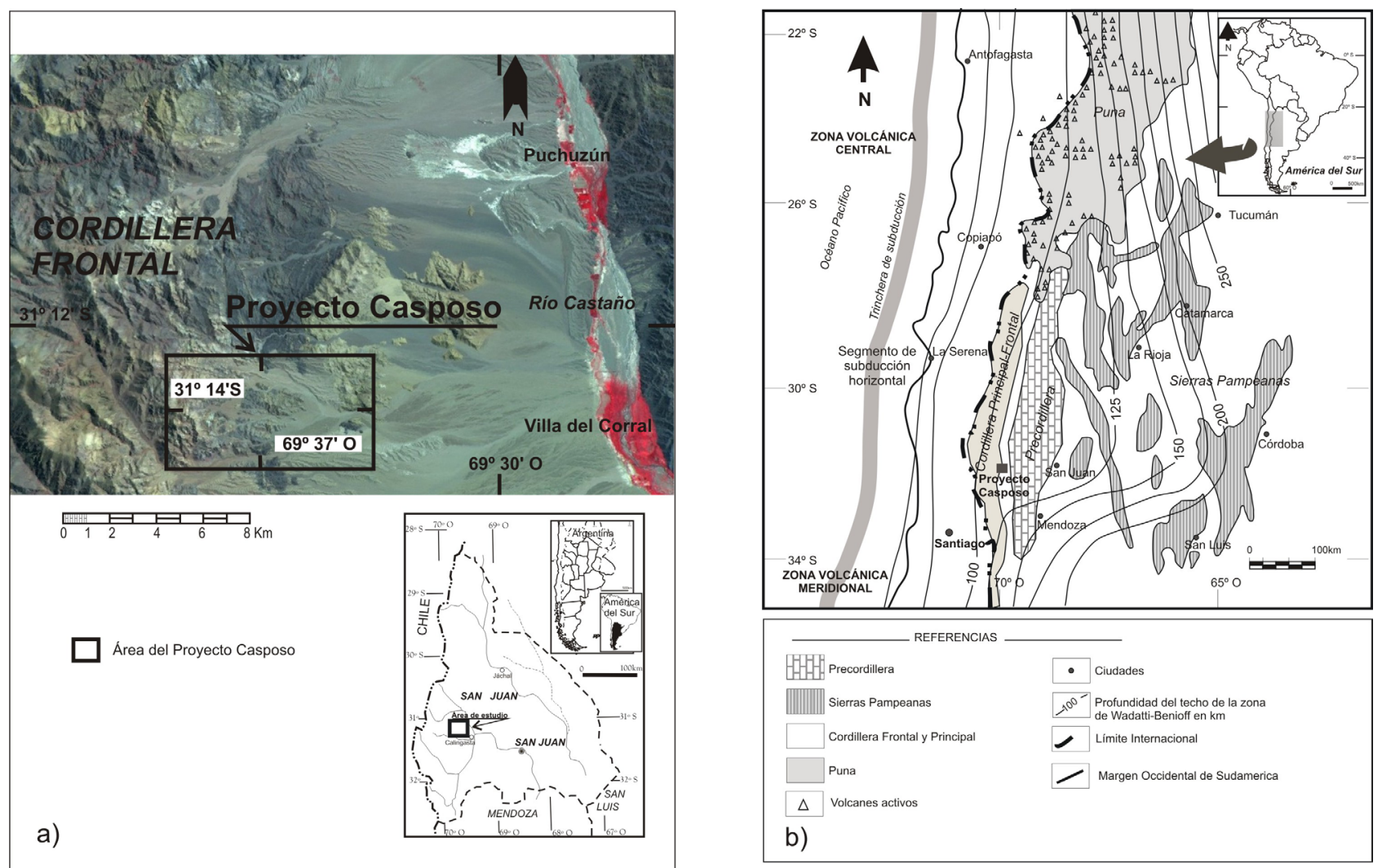

Figura 1. Ubicación geográfica del Proyecto Casposo, provincia de San Juan. Argentina. Marco tectónico de la región. 
sismológicos han demostrado la existencia de una fragmentación en el ángulo de subducción de la placa de Nazca, definiendo segmentos latitudinales, que cambian de "subducción normal" (aproximadamente $30^{\circ}$ ) a ángulos de subducción subhorizontal $\left(<14^{\circ}\right)$ (Barazanghi e Isacks, 1976), a una tasa de $6.3 \mathrm{~cm} /$ año (Jordan et al., 1983; Smalley e Isacks, 1987, 1990; Smalley et al., 1993, Alvarado y Beck, 2006) (Figura 1). La distribución de hipocentros a lo largo de la costa chilena muestra grandes diferencias en la sismicidad, que disminuye notablemente de Norte a Sur y de Oeste a Este. La región ubicada entre los $28^{\circ}$ y $36^{\circ} \mathrm{S}$ se caracteriza por tener una elevada sismicidad y una activa historia de terremotos.

La información geológica también ha mostrado que las inhomogeneidades en el ángulo de subducción tienen una relación directa con el estilo tectónico y entorno geológico de la placa Sudamericana (Jordan et al., 1983). La interacción de placas define un segmento de subducción horizontal entre los $27^{\circ}$ y $33^{\circ}$ de latitud, que determina diferentes características tectónicas y morfológicas respecto a los segmentos ubicados al norte y al sur del mismo (Barazanghi e Isacks, 1976, Jordan et al.,1983) y en el que se concentran aproximadamente el $80 \%$ de las deformaciones cuaternarias actualmente conocidas en el territorio argentino (Costa et al., 2000). En esta región, la placa de Nazca subduce a aproximadamente $100 \mathrm{~km}$ de profundidad y luego se extiende horizontalmente por varios cientos de kilómetros hacia el Este hasta descender en el manto. Esa horizontalización de la placa se habría iniciado hace 8-10 Ma (Jordan y Gardeweg, 1989; Kay et al.,1991). Este proceso también se asocia con la ausencia de un arco volcánico entre esas latitudes, la migración de la Precordillera hacia el Este y el ascenso del basamento de Sierras Pampeanas.

En la provincia de San Juan, una sección vertical tipo muestra una distribución bimodal de la sismicidad. Los eventos de profundidad intermedia corresponden a la sismicidad de intraplaca a lo largo de la zona de WadatiBenioff de la placa de Nazca subductada (90-120 km). Los eventos superficiales corresponden a deformaciones corticales localizadas en la parte superior de la placa (5-35 $\mathrm{km})$. Otra zona de baja actividad sísmica aparece también entre 40 y 95 km. (INPRES, 1982; Smalley e Isacks, 1987).

En el valle de Calingasta se aprecia una importante actividad sísmica entre los 100 y $200 \mathrm{~km}$ de profundidad. Estos eventos están asociados con deformaciones que se producen tanto en el interior como en la parte superior de la placa de Nazca, en su movimiento de subducción al Este.

\section{Deformación cuaternaria}

Debido a la migración hacia el Este del frente orogénico Andino durante el Neógeno, entre los $27^{\circ}$ y $32^{\circ} \mathrm{S}$, la actividad orogénica durante el Plioceno-Pleistoceno se manifestó por el ascenso de la faja plegada y corrida de
Precordillera (Ramos, 1988). Las principales estructuras cuaternarias se ubican principalmente entre Precordillera Oriental y la porción occidental de las sierras Pampeanas (Cortés et al., 1999; Costa et al., 2000; Costa et al., 2006).

$\mathrm{Al}$ analizar el marco tectónico neógeno y actual de la región de estudio se identificaron las fuentes de actividad sísmica presentes con algunos parámetros característicos de cada una de ellas, a los fines de establecer si existía una correspondencia con la evidencia neotectónica (Figura 2). Muchas veces estas fallas superficiales de intraplaca han sido subestimadas como fuentes sismogénicas, sin embargo, estudios recientes han sugerido que terremotos prehistóricos han originado rupturas en superficie y escarpes cosísimicos de más de $1 \mathrm{~m}$ (Costa et al., 2001; Costa y Vita Finzi, 1996; Costa et al., 2006).

La falla El Tigre se ubica en el piedemonte occidental de la Precordillera, al este del Proyecto Casposo. Esta falla es dextral, con componente normal con el plano que inclina $60^{\circ}$ al Este, el escarpe posee el bloque elevado al Oeste, con alturas que varían entre 0.80 y $50 \mathrm{~m}$ y el desplazamiento máximo lateral derecho es de $280 \mathrm{~m}$, medido en unidades cuaternarias. Bastías (1986) estimó un sismo máximo probable de magnitud $\mathrm{M}=7.5 \mathrm{y}$ una tasa de recurrencia de entre 500 y 2000 años. Siame et al. (1997) estudiaron el segmento de la falla El Tigre ubicado entre los ríos Jáchal y San Juan, en un tramo de alrededor a $120 \mathrm{~km}$ donde la falla afecta seis niveles de abanicos aluviales cuyas edades, obtenidas por dataciones cosmogénicas varían entre menos de 700000 años y 45000 años para las superficies más recientes. Determinaron un desplazamiento lateral derecho de aproximadamente $150 \pm 20 \mathrm{~m}$ y un máximo desplazamiento de $260 \pm 20 \mathrm{~m}$. Calcularon un límite inferior en la tasa de deslizamiento de $0.5 \mathrm{~mm} / \mathrm{año} \mathrm{y}$ un límite superior de $5 \mathrm{~mm} / \mathrm{año}$. En un ambiente cuya tasa de erosión es baja, la falla puede exhibir rasgos geomórficos bien preservados y tener una tasa de deslizamiento relativamente más baja que la determinada por Bastías (1986) y Bastías et al. (1990).

Costa et al. (2000) mencionan un ramal paralelo a la falla El Tigre denominado falla Cántaro de Oro con una longitud aproximada de $25 \mathrm{~km}$.

Escasos kilómetros al este del área de proyecto se ubica la falla Villa Corral de rumbo N-S, afecta depósitos del Pleistoceno. Esta falla tiene una longitud aproximada de 5 $\mathrm{km}$ y su escarpe, a contrapendiente, mide un máximo de 10 $\mathrm{m}$. La tasa de deslizamiento se estimó en $0.01 \mathrm{~mm} /$ año y la magnitud en Ms 5.9. En la porción distal del abanico del río Castaño, se ubica una falla inversa de rumbo N-S y longitud aproximada a $10 \mathrm{~km}$ que inclina $30-45^{\circ}$ al Este y que coloca depósitos de edad terciaria sobre los fanglomerados del Pleistoceno. En este trabajo se la denomina falla abanico del Río Castaño.

Al sur del río San Juan los principales rasgos neotectónicos en el valle de Calingasta se localizan en el piedemonte occidental de Precordillera Occidental. Por su proximidad al área de sitio del Proyecto Casposo, se 

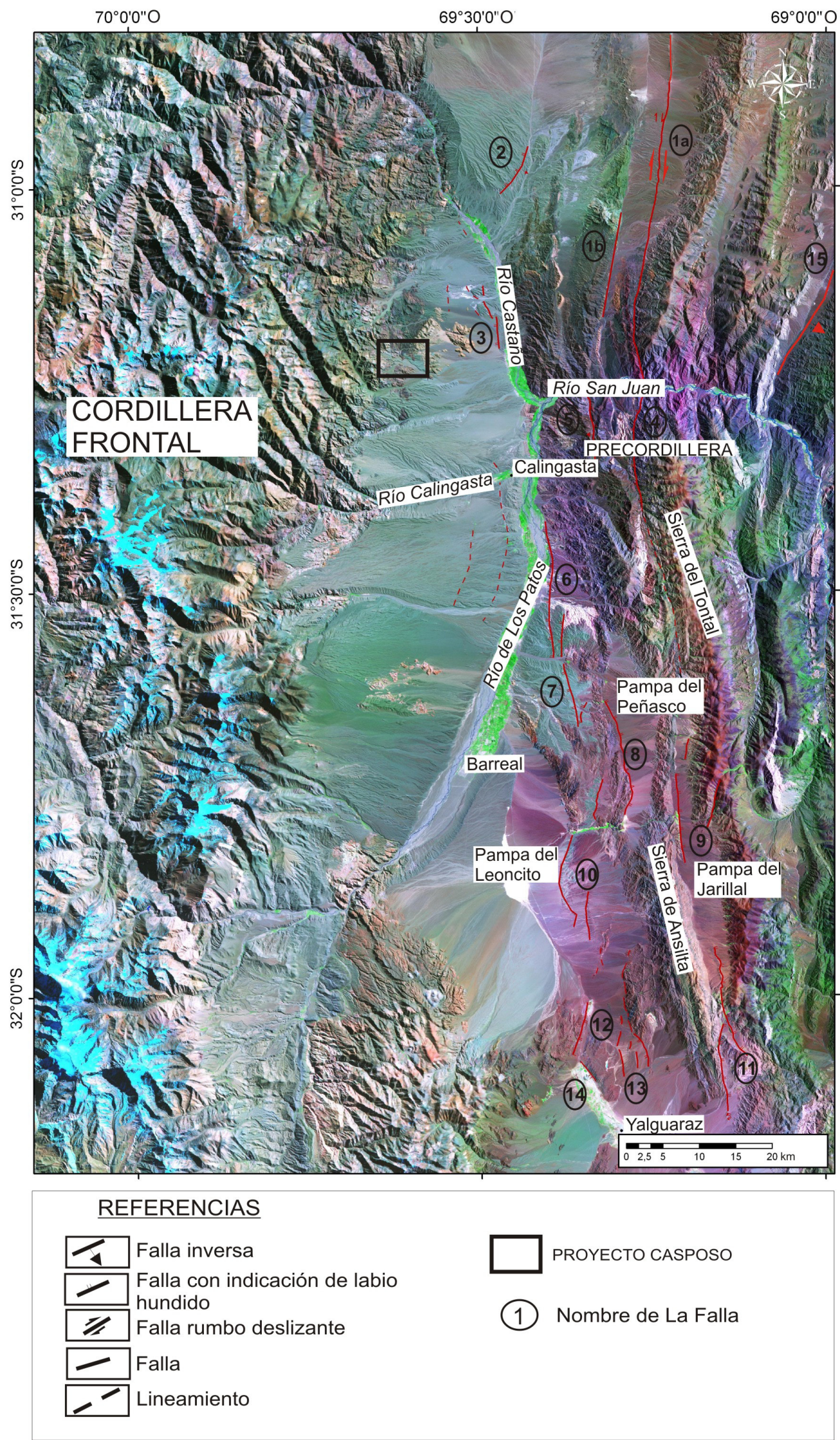

Figura 2. Fallas con actividad tectónica cuaternaria: 1a. El Tigre, 1b. Cántaro de Oro; 2. Río Castaño; 3. Villa Corral; 4. Tontal; 5. Carrizal; 6. Alcázar; 7. Mosquitos; 8. Cepeda; 9. La Horqueta; 10. Barreal-Las Peñas; 11. Ansilta; 12. Lomas Bayas; 13. Puesto Tapia; 14. Yalguaraz; 15. La Cantera. 
describen a continuación (Figura 2).

En el sector de cordones del interior precordillerano se encuentra la falla inversa El Carrizal, con aproximadamente $20 \mathrm{~km}$ de longitud y un rumbo promedio de $\mathrm{N} 14^{\circ} \mathrm{O} \pm 16^{\circ}$, que inclina $50^{\circ}$ al Oeste. Parte de su traza sobrepone rocas ordovícicas a sedimentos cuaternarios. La edad del último movimiento corresponde al Cuaternario $(<1.6 \mathrm{Ma})$ (Costa et al., 2000).

La falla Tontal es inversa con inclinación al este. La longitud de esta falla en sedimentos cuaternarios es de aproximadamente $7 \mathrm{~km}$ con un rumbo promedio $\mathrm{N} 8^{\circ} \mathrm{O} \pm 7^{\circ}$. Las alturas máximas de los escarpes varían entre 36 y $40 \mathrm{~m}$ en su tramo centro y sur. Si se considera que los depósitos afectados por la falla son contemporáneos a los datados y afectados por la falla El Tigre, se puede inferir un desplazamiento de la falla entre $0.05-0.057 \mathrm{~mm} / \mathrm{año}$, que según la clasificación de Slemmons (1982), corresponde a una falla Tipo B, con moderada actividad durante el Cuaternario. Paredes (1993) determinó una actividad tectónica moderada para la falla en su segmento austral.

La falla La Horqueta, posiblemente inversa y oblicua al frente montañoso, posee unos $7 \mathrm{~km}$ de longitud y un rumbo promedio $\mathrm{N} 34^{\circ} \mathrm{E} \pm 7^{\circ}$, que inclinaría al oeste. Esta falla originó un escarpe con ladera al este en los abanicos pedemontanos más antiguos. La falla termina al sur en una falla oblicua (falla Corrales de Araya) que se extiende con rumbo nornoroeste con un escarpe de falla con ladera al este que corresponde a la falla Jarillal (Bastías et al., 1990), la cual asciende hacia el este sedimentitas triásicas sobre depósitos aluviales cuaternarios.

La falla Jarillal-Ansilta es inversa y posee una longitud aproximada de $17 \mathrm{~km}$ con rumbo promedio de $\mathrm{N} 9^{\circ} \mathrm{E} \pm 21^{\circ}$, inclina al oeste y el bloque hundido se ubica al este. La altura de los escarpes varía entre 10 y $16 \mathrm{~m}$. La edad estimada del último movimiento para estas fallas es Cuaternaria $(\sim 700$ $\mathrm{Ma}$ ). La tasa de deslizamiento para esta falla se ubicaría en el rango entre 0.01-0.02 mm/año. Esto implica, según Slemmons (1982), una falla de Tipo B, con moderada actividad, con rasgos geomorfológicos bien preservados.

Quartino et al. (1971) señalan numerosas fallas que cortan depósitos de edad cuaternaria como la falla El Alcázar, inversa y que afecta abanicos aluviales cuaternarios. Dicha falla se extendería por aproximadamente $15 \mathrm{~km}$ con rumbo general $\mathrm{N} 5^{\circ} \mathrm{O} \pm 29^{\circ}$ y con el bloque oeste hundido. La tasa de movimiento para estas fallas se desconoce y se considera que la edad del último movimiento es inferior a 1.6 Ma.

La falla Mosquitos es inversa con inclinación promedio $65^{\circ} \mathrm{SE}$ y sobrepone depósitos triásicos sobre el Cuaternario. Esta falla se extiende a lo largo de $11 \mathrm{~km}$ con un rumbo submeridianal.

La falla Cepeda es inversa y con inclinación al oeste en un ángulo no especificado, corta los sedimentos aluviales cuaternarios contiguos al frente generando un notorio escarpe con ladera al este. Esta falla posee una longitud aproximada de $13 \mathrm{~km}$ con un rumbo promedio $5^{\circ} \mathrm{O} \pm 28^{\circ}$.

Cortés y Cegarra (2004) describen en el extremo norte de la Precordillera Sur, un extenso cinturón transpresivo cenozoico, denominado Barreal-Las Peñas de $150 \mathrm{~km}$ de longitud de orientación NNO constituido por bloques montañosos escalonados delimitados por fallas inversas y zonas de cizalla con componentes de desplazamiento de rumbo sinestral. La faja muestra una alta concentración y variedad de evidencias de actividad tectónica pleistocena y holocena, representadas por escarpes pedemontanos, pliegues de crecimiento y frentes montañosos reactivados y segmentados. Al sur del valle de Calingasta se ubica el Grupo de Falla Yalguaraz. Se trata de estructuras que marginan parcialmente el flanco oriental de la Cordillera Frontal y que afectan además las serranías menores en la pampa de Yalguaraz, un bolsón intermontano que separa Cordillera de Precordillera. Cortés (1998) señala una longitud de falla de aproximadamente $25 \mathrm{~km}$, con un rumbo promedio de $\mathrm{N} 17^{\circ} \pm 23^{\circ}$. Son fallas inversas, que inclinan $35^{\circ}$ al oeste. La expresión geomorfológica más notable son los escarpes discontinuos en abanicos aluviales y el trazo rectilíneo de la unión serranía-piedemonte.

La falla Puesto Tapia de rumbo NNO y N-S corta abanicos aluviales holocenos y la falla Lomas Bayas inclina $58^{\circ}$ al suroeste y posee un azimut de $310-315^{\circ}$, junto a la misma, los estratos cuaternarios presentan un pliegue de arrastre con inclinaciones de $50^{\circ}$ al suroeste, debido al desplazamiento normal a lo largo de la misma (Cortés y Costa, 1993). Si se considera una edad aproximada de 700000 años para los depósitos afectados por esta estructura, la tasa de deslizamiento de la falla se encontraría entre 0.01$0.02 \mathrm{~m} / \mathrm{año}$, es decir que es una falla Tipo B, con moderada actividad tectónica durante el Cuaternario.

En el faldeo oriental de Cordillera Frontal, las evidencias estructurales de actividad tectónica durante el Cuaternario son escasas. En la porción norte de la provincia de Mendoza se ubica la falla Cordón Cucaracha, que hacia el norte genera un escarpe de $2 \mathrm{~m}$ con el bloque hundido al este. Esta falla posee una longitud aproximada de $10 \mathrm{~km}$ y un rumbo NE-SO.

La falla La Cantera se encuentra al norte del río San Juan y posee evidencias de actividad cuaternaria muy notorias. Su longitud es de $21.5 \mathrm{~km}$ con buenos indicios de actividad holocena. Es una falla inversa que inclina al oeste entre $15^{\circ}$ y $60^{\circ}$. Mingorance (1998) asigna a esta falla el sismo histórico de 1924, (Ms 6.0). El rumbo de la falla es N $8^{\circ} \mathrm{E}$ al norte y $\mathrm{N} 27^{\circ} \mathrm{E}$ al sur. La altura total del escarpe es de $14 \mathrm{~m}$ y la tasa de deslizamiento de aproximadamente 0.35 $\mathrm{mm} /$ año (moderada actividad). El sismo máximo para esta falla se estima en Ms 6.6.

En la Tabla 1 se resumen las fuentes sismogénicas descritas en los párrafos anteriores.

\section{Análisis determinístico de la amenaza sísmica}

Sobre la base de la información neotectónica recopilada se realizó una evaluación determinística de la amenaza 
sísmica en el sitio de emplazamiento del Proyecto Casposo, considerando la situación más desfavorable (magnitud máxima/distancia mínima) para cada una de las fuentes sismogénicas identificadas en la Tabla 1. Se incluyó además, la zona de Benioff como una fuente sismogénica independiente, para la cual se asumió una magnitud máxima Ms 7.5 y cuyo evento más desfavorable sería un terremoto con hipocentro bajo el sitio de proyecto y a una profundidad mínima de $80 \mathrm{~km}$.

Para estimar el movimiento sísmico que provocarían los eventos considerados en cada una de las fuentes, se utilizaron las leyes de atenuación propuestas por Abrahamson y Silva (1997) para terremotos de corteza y la de Youngs et al. (1997) para los terremotos de subducción (zona de Benioff). Las dos relaciones de atenuación utilizadas permiten estimar el espectro de respuesta de pseudoaceleración para el $5 \%$ de amortiguamiento, en función de la magnitud del evento, la distancia mínima a la ruptura y el tipo de falla. Los espectros obtenidos corresponden a la base rocosa. En el caso de terremotos originados en la corteza y fallas inversas, también se diferencia entre sitios ubicados en el bloque ascendente o descendente. Además, en los casos en que corresponde, se han tenido en cuenta los efectos de directividad mediante el modelo de Sommerville et al. (1997). Por otra parte, se consideró que los terremotos originados en la zona de Benioff son eventos intraplaca, es decir, que ocurren dentro de la placa subducida por fallamiento normal.

La Figura 3 muestra los espectros de pseudoaceleración para un amortiguamiento del $5 \%$, obtenidos para los cuatro eventos que controlan la amenaza. Un sismo de $\mathrm{M}=8$ en la falla El Tigre, a una distancia mínima de $34 \mathrm{~km}$, controla la amenaza para los periodos largos. Para los periodos cortos e intermedios, la amenaza resulta controlada por alguno de los eventos que se indican en la Figura 3, dependiendo del número de desviaciones estándar que se considere (media, media más una desviación estándar, etc.).

Evidentemente, además de las fuentes sismogénicas consideradas, existe la posibilidad de que un terremoto de magnitud importante (6-6.5) ocurra a una distancia menor del proyecto de la que se encuentra la falla de Villa Corral $(16 \mathrm{~km})$. Apoya esta hipótesis el hecho de que en la zona de la Cordillera Frontal los catálogos de sismicidad instrumental incluyan un número de sismos con magnitudes de ese orden, tal como se verá en un apartado posterior. La peor situación ocurriría con un terremoto con hipocentro inmediatamente debajo del sitio del proyecto. Se intuye que esta situación es extremadamente improbable, pero no se dispone de un criterio lógico para especificar una distancia de un evento de esas características, sin recurrir a un análisis probabilístico.

\section{Criterios de verificación sísmica de las obras}

Ante la ausencia en Argentina de una norma aplicable a obras no edilicias, se ha propuesto un criterio de verificación acorde con la práctica actual a nivel mundial. En primer lugar se clasifican las obras en dos grandes grupos. Se consideran obras críticas, a aquellas cuyo colapso constituye una amenaza para la seguridad pública y/o suponen un riesgo para uno o más centros poblados o para el medio ambiente. Se encuadran dentro de esta categoría las presas de colas, presas de embalse o estructuras de

Tabla 1. Fuentes sismogénicas relevantes para el proyecto, identificadas a partir del estudio de neotectónica: (1) Longitud total de la falla o sistema de fallas, (2) Distancia mínima entre la traza de la falla y el proyecto (en superficie) (3) Rumbo promedio de la traza de falla y buzamiento del plano de falla, (4) Distancia mínima entre el plano de ruptura y el sitio del proyecto, (5) Máxima longitud de ruptura esperada, (6) Magnitud Media y Media + 1 desviación estándar obtenida a partir de la correlación de Wells y Coppersmith (1994) con la longitud de ruptura en superficie, (7) Magnitud momento máxima asignada a cada falla en base a los rasgos neotectónicos y la sismicidad histórica, (8) Tasas de deslizamiento estimadas.

\begin{tabular}{|c|c|c|c|c|c|c|c|c|c|c|c|c|}
\hline No & Fuente & Tipo & $\begin{array}{l}\text { Long Total } \\
{[\mathrm{km}]} \\
(1)\end{array}$ & $\begin{array}{c}\mathrm{r} \\
{[\mathrm{km}]} \\
(2)\end{array}$ & $\begin{array}{l}\mathrm{R} \\
(3)\end{array}$ & $\begin{array}{l}\mathrm{Buz} \\
(3)\end{array}$ & $\begin{array}{c}\mathrm{r}_{\text {rup }} \\
{[\mathrm{km}]} \\
(4)\end{array}$ & $\begin{array}{l}\mathrm{L}_{\text {rup }} \\
{[\mathrm{km}]} \\
(5)\end{array}$ & $\begin{array}{l}M \\
(6)\end{array}$ & $\begin{array}{c}M+1 \sigma \\
(6)\end{array}$ & $\begin{array}{l}M_{w} \\
(7)\end{array}$ & $\begin{array}{c}\text { IR } \\
{[\mathrm{mm} / \mathrm{año}]} \\
(8)\end{array}$ \\
\hline 1 & El Tigre & $\begin{array}{c}\text { Normal } \\
\text { Rumbo dextral }\end{array}$ & $>200$ & 34 & $\mathrm{~N} 9^{\circ} \mathrm{E}$ & $80^{\circ} \mathrm{E}$ & 34 & 153 & 7.6 & 7.9 & 8 & 1.0 a 5.0 \\
\hline 2 & La Cantera & Inversa & 47 & 52 & $\mathrm{~N} 20^{\circ} \mathrm{E}$ & $45^{\circ} \mathrm{O}$ & 37 & 25 & 6.7 & 7 & 7 & 0.35 \\
\hline 3 & $\begin{array}{l}\text { Sistema Maradona - } \\
\text { La Dehesa }\end{array}$ & Inversa & 150 & 72 & $\mathrm{~N} 6^{\circ} \mathrm{E}$ & $35^{\circ} \mathrm{O}$ & 41 & 20 & 6.6 & 6.9 & 6.9 & 0.01 a 0.03 \\
\hline 4 & Falla Carrizal & Inversa & 24 & 28 & $\mathrm{~N} 14^{\circ} \mathrm{O}$ & $50^{\circ} \mathrm{O}$ & 22 & 20 & 6.6 & 6.9 & 6.9 & \\
\hline 5 & $\begin{array}{l}\text { Grupo Tontal - } \\
\text { Horqueta - Jarillal - } \\
\text { Ansilta }\end{array}$ & Inversa & 103 & 35 & $\mathrm{~N} 8^{\circ} \mathrm{O}$ & $>70^{\circ} \mathrm{E}$ & 35 & 17 & 6.5 & 6.8 & 6.8 & 0.01 a 0.02 \\
\hline 6 & $\begin{array}{l}\text { Sistema Alcazar- } \\
\text { Mosquitos }\end{array}$ & Inversa & 29 & 31 & $\mathrm{~N} 5^{\circ} \mathrm{O}$ & $65^{\circ} \mathrm{E}$ & 33 & 11 & 6.3 & 6.6 & 6.6 & \\
\hline 7 & Falla Cepeda & Inversa & 13 & 56 & $\mathrm{~N} 15^{\circ} \mathrm{O}$ & $45^{\circ} \mathrm{O} ?$ & 55 & 13 & 6.4 & 6.6 & 6.7 & \\
\hline 8 & Grupo Yalguaraz & Inversa & 25 & 88 & $\mathrm{~N} 0^{\circ}$ & $35^{\circ} \mathrm{O}$ & 85 & 25 & 6.7 & 7 & 7 & 0.01 a 0.02 \\
\hline 9 & Falla Villa Corral & Inversa? & 5 & 15 & $\mathrm{~N} 0^{\circ}$ & $\mathrm{E}$ ? & 16 & 5 & 5.9 & 6.1 & 6.1 & 0.01 \\
\hline 10 & Falla Castaño & Inversa & 10 & 27 & $\mathrm{~N} 32^{\circ} \mathrm{E}$ & $40^{\circ} \mathrm{E}$ & 28 & 10 & 6.2 & 6.5 & 6.5 & \\
\hline
\end{tabular}




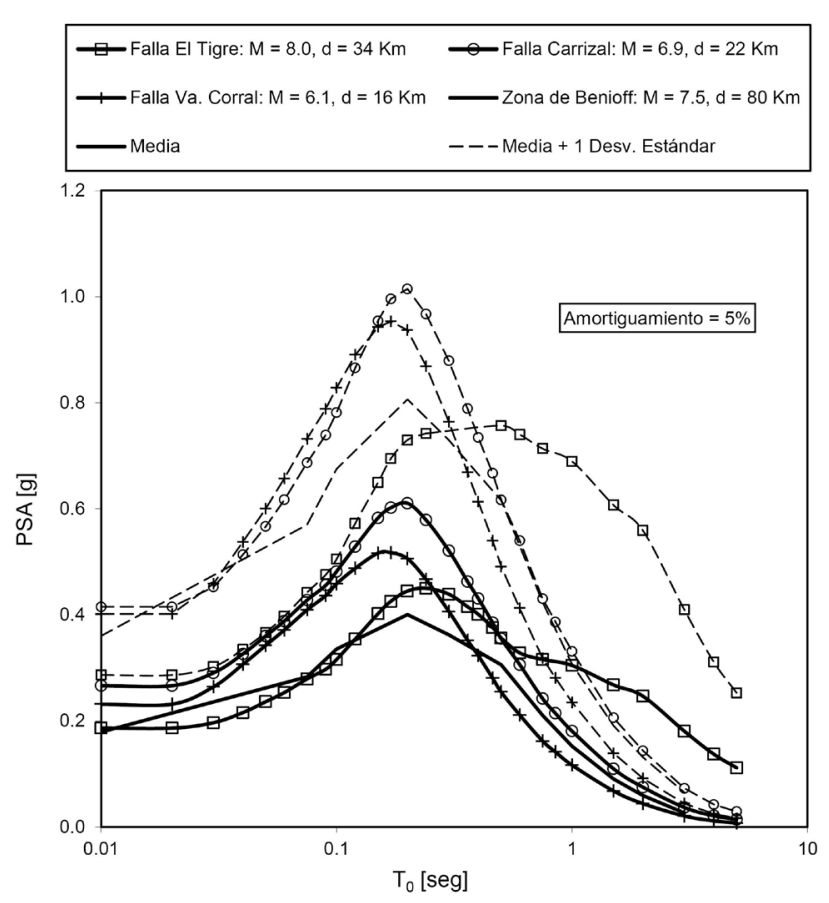

Figura 3. Espectros de respuesta de pseudoaceleración para el $5 \%$ de amortiguamiento, calculado para todos los eventos identificados en el análisis determinístico de la amenaza. La escala es lineal en el eje de las ordenadas y logarítmica en el eje de las abscisas. Las líneas punteadas corresponden a valores "medios +1 Desv. Estándar" y las líneas enteras a valores "medios".

almacenamiento de substancias contaminantes cuyo vertido pueda provocar daños irreversibles al medio ambiente. Todas aquellas obras que no se encuadran en la categoría de críticas se consideran no críticas.

La extensión en el tiempo para el Proyecto Casposo se prevé en 6 años. Con el fin de contar con un margen de seguridad por eventuales demoras o extensiones en la operación, se adoptó un tiempo de vida del proyecto de 10 años. Por otra parte, se prevé que los residuos (escombreras y pila de colas) permanezcan en el sitio a perpetuidad. Sobre la base de la práctica de ingeniería a nivel mundial (FEMA, 2000; ICOLD, 1989), se propuso para el Proyecto Casposo un esquema de verificación en dos niveles, que se ilustra en la Tabla 2. Se establece un Terremoto de Operación Normal (TON) con un $50 \%$ de probabilidad de ser excedido en la vida útil de las obras, para el cual todas las obras deben tener sólo daños mínimos, cuya reparación no requiera una parada en la operación del proyecto. Para estructuras no críticas, se establece además un Terremoto Máximo de Diseño (TMD), con una probabilidad de excedencia del 1 $\%$ en la vida útil de las obras. El objetivo de diseño en este caso es evitar el colapso para preservar la seguridad de las personas que trabajan en el proyecto.

El Proyecto Casposo no contempla la construcción de obras críticas tales como presas de colas (se prevé el uso de la técnica de secado de las colas por filtrado y almacenamiento seco o dry-stacking). Sin embargo, se estableció un criterio
Tabla 2. Criterios de verificación sísmica para el Proyecto Casposo.

\begin{tabular}{|c|c|c|c|c|}
\hline Tipo de Obra & $\begin{array}{l}\text { Probabilidad } \\
\text { de excedencia }\end{array}$ & $\begin{array}{l}\text { Periodo de } \\
\text { retorno }\end{array}$ & Objetivo de diseño & $\begin{array}{c}\text { Designación del } \\
\text { movimiento sísmico } \\
\text { de diseño }\end{array}$ \\
\hline TODAS & $\begin{array}{c}50 \% \text { en } 10 \\
\text { años }\end{array}$ & 15 años & $\begin{array}{l}\text { Sin daños } \\
\text { estructurales. } \\
\text { Mínima interrupción } \\
\text { de la operación }\end{array}$ & TON \\
\hline NO CRÍTICA & $1 \%$ en 10 años & 1000 años & $\begin{array}{l}\text { Seguridad de vida de } \\
\text { las personas que } \\
\text { trabajan en el } \\
\text { proyecto }\end{array}$ & $\mathrm{TMD}_{1000}$ \\
\hline CRÍTICA & $1 \%$ en 100 & 10000 años & $\begin{array}{l}\text { Seguridad pública. } \\
\text { Seguridad de vida y } \\
\text { bienes de la } \\
\text { población. } \\
\text { Se aceptan daños, sin } \\
\text { colapso, que no } \\
\text { provoquen escapes } \\
\text { incontrolados de } \\
\text { agua, sólidos o } \\
\text { substancias } \\
\text { contaminantes. }\end{array}$ & $\mathrm{TMD}_{10000}$ \\
\hline
\end{tabular}

de verificación para eventuales obras críticas. Dicho criterio implica la verificación con un movimiento sísmico con una probabilidad de excedencia del $1 \%$ en 100 años (periodo de retorno de 10000 años). El objetivo fundamental de diseño en este caso sería el resguardo de la seguridad pública, tanto en lo que hace a la vida y bienes de la población, como a la preservación del medio ambiente y los recursos naturales (por ejemplo, para una presa de colas se aceptarían daños, sin colapso, que no provoquen escapes incontrolados de agua, sólidos o substancias contaminantes).

\section{Análisis de la información sismológica}

Se compiló un catálogo de sismos para el área rectangular comprendida entre los $29^{\circ} 45^{\prime}$ y $32^{\circ} 30^{\prime} \mathrm{N}$ y los $68^{\circ} 20^{\prime}$ y $70^{\circ} 50^{\prime} \mathrm{W}$, incluyendo tanto sismos históricos como instrumentales. El catálogo resultante fue procesado para eliminar las réplicas.

Salvo algunas pocas excepciones, hasta el momento no es posible establecer la relación entre la actividad sísmica histórica e instrumental y los segmentos de falla con señales de actividad cuaternaria conocidos en la región. Puesto que con la información disponible es imposible establecer la ley de frecuencia de excedencia de magnitud para cada falla identificada, se ha procesado la información sismológica dividiéndola en tres zonas dentro de las cuales el estilo sismotectónico puede asumirse como uniforme. Según la ubicación del foco de cada evento, se subdividió el catálogo en las siguientes zonas: Precordillera (al este del meridiano $69^{\circ} 24^{\prime}$ y profundidad entre 0 y $70 \mathrm{~km}$ ), Cordillera Frontal (al oeste del meridiano $69^{\circ} 24^{\prime}$ y profundidad entre 0 y 70 $\mathrm{km}$ ), Zona de Benioff (profundidad entre 70-150 km). Los epicentros de estos eventos se indican en las Figuras 4 y 5.

Es esperable que los catálogos obtenidos para cada una 


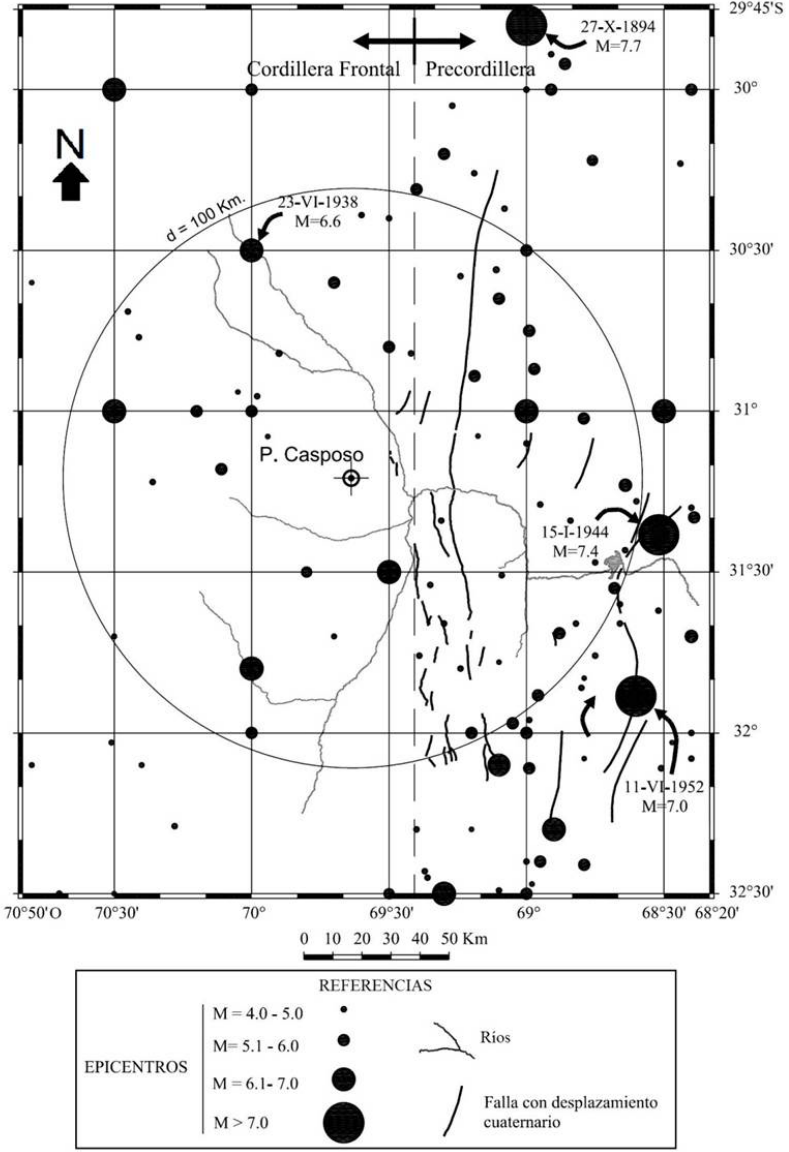

Figura 4. Epicentros de todos los eventos obtenidos del catálogo sísmico de Precordillera y Cordillera Frontal (Profundidad focal $<70 \mathrm{~km}$ ).

de las tres zonas estudiadas no estén completos para todas las magnitudes en un periodo de tiempo lo suficientemente largo, como para ser representativos de la actividad sísmica de la zona. Por ello se han tomado ventanas de tiempo de extensión variable para diferentes rangos de magnitud. Se asume que todos los eventos de magnitud mayor o igual a 7 han quedado registrados en documentos históricos a partir de la colonización española (a partir del 1600). Con criterio similar, todos los de magnitud mayor a 6 se han identificado a partir de 1922, año en que comenzó a operar el observatorio sismológico de la ciudad de La Plata. Teniendo en cuenta el paulatino mejoramiento de la red sismológica mundial que ha permitido aumentar progresivamente la capacidad de captar y ubicar eventos sísmicos cada vez menores, se supone que todos los eventos mayores que $5 \mathrm{se}$ han registrado a partir de 1960, todos los eventos mayores que 4.5 desde 1985 y todos los eventos de magnitud mayor o igual a 4 desde 1995.

Para cada una de las tres zonas estudiadas se ajustó una ley de Gutemberg-Richter (G-R), de tipo acotada (Figura 6):

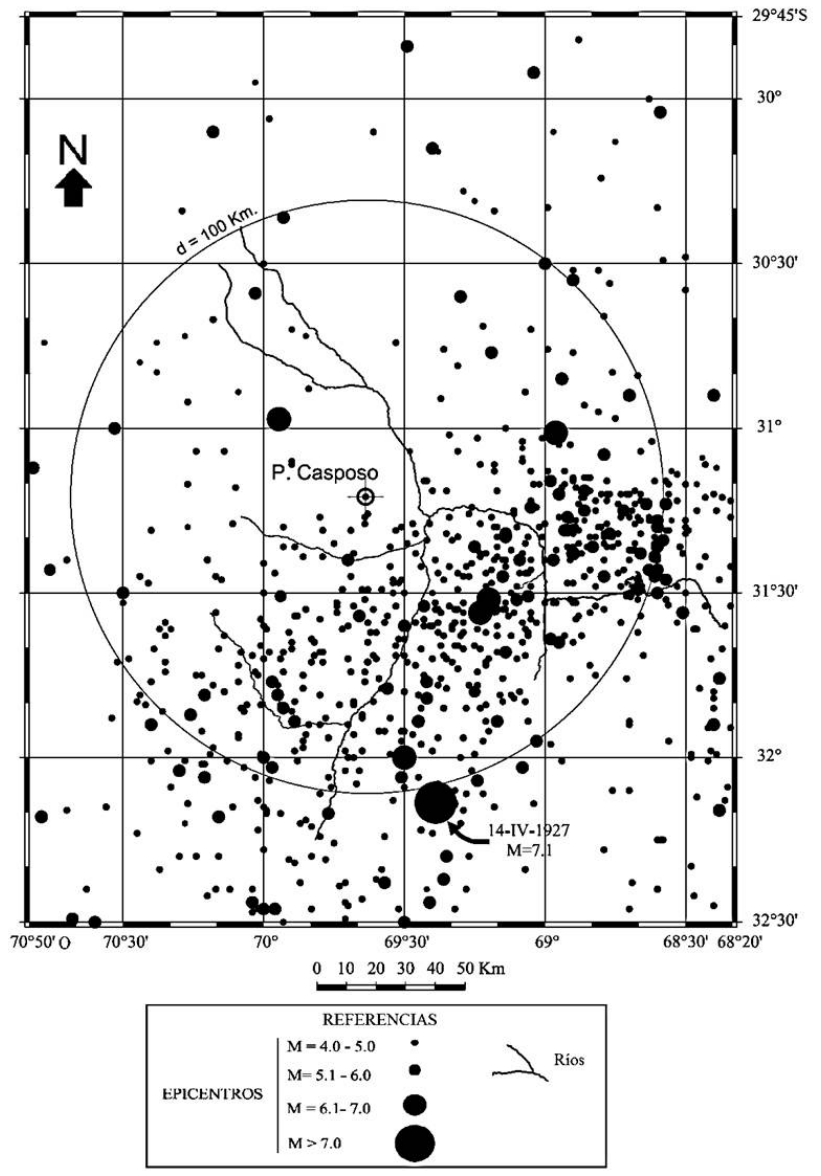

Figura 5. Epicentros de todos los eventos del catálogo sísmico de la zona de Benioff (Profundidad Focal $>70 \mathrm{~km}$ ).

$$
\lambda_{m}=v \frac{\exp \left[\beta\left(m-m_{0}\right)\right]-\exp \left[\beta\left(m_{\max }-m_{0}\right)\right]}{1-\exp \left[\beta\left(m_{\max }-m_{0}\right)\right]}
$$

donde :

$\lambda_{m} \quad=$ frecuencia anual de excedencia de la magnitud $m$

$\mathrm{m} \quad=$ magnitud

$\mathrm{m}_{0}=$ magnitud mínima considerada en el ajuste

$\mathrm{m}_{\max }=$ magnitud máxima asumida para la fuente

$v^{\max }=\exp \left(\alpha-\beta . m_{0}\right)=$ frecuencia anual de excedencia de la magnitud mínima

$\alpha \quad=2.303 \times a$

$\beta \quad=2.303 \times b$

$a$ y $b$ son los parámetros de la Ley de Gutemberg-Richter estándar.

Se adoptó como magnitud mínima $\mathrm{m}_{0}=4.5$, para las dos fuentes sismogénicas de corteza y $\mathrm{m}_{0}=5$, para la zona de Benioff. La magnitud máxima se estimó para cada fuente en base a la información de neotectónica disponible, fundamentalmente mediante correlaciones con la longitud de ruptura (ver Tabla 1). Los parámetros a y b de la ley (G-R) se obtuvieron por ajuste de los datos sismológicos (Tabla 3). 


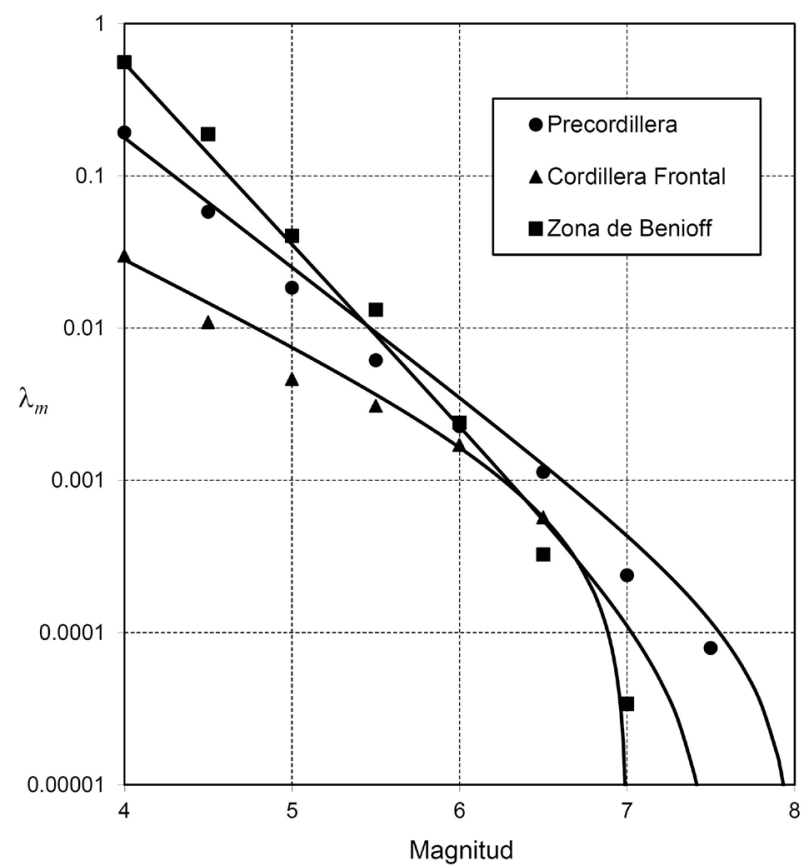

Figura 6. Ajuste de Gutemberg-Richter para las tres fuentes sismogénicas consideradas en el análisis probabilístico. La escala es lineal en el eje de las abscisas y logarítmica en el eje de las ordenadas.

Tabla 3. Parámetros de la Ley Gutemberg-Richter obtenidos por ajuste de los catálogos de sismos.

\begin{tabular}{cccc}
\hline \multirow{2}{*}{ FUENTE } & $\begin{array}{c}\text { 1) } \\
\text { PRECORDILLERA }\end{array}$ & $\begin{array}{c}\text { 2) CORDILLERA } \\
\text { FRONTAL }\end{array}$ & $\begin{array}{c}\text { 3) ZONA DE } \\
\text { BENIOFF }\end{array}$ \\
\hline$m_{0}$ & 4.5 & 4.5 & 5 \\
$m_{\max }$ & 8 & 7 & 7.5 \\
$A$ & 2.65 & 0.64 & 4.5 \\
$B$ & 0.85 & 0.55 & 1.19 \\
$v$ & $6.68 \times 10^{-2}$ & $1.46 \times 10^{-2}$ & $3.55 \times 10^{-2}$ \\
\hline
\end{tabular}

\section{Análisis probabilístico de la amenaza}

Se llevó a cabo un análisis probabilístico de la amenaza sísmica, considerando como fuentes sismogénicas las tres zonas definidas en el apartado anterior, caracterizadas por los parámetros resumidos en la Tabla 3. Se utilizó la relación de Abrahamson y Silva (1997) para los terremotos de corteza (Precordillera y Cordillera Frontal) y la de Youngs et al. (1997) para la Zona de Benioff. Se consideró que el sitio de emplazamiento del proyecto es roca o suelo duro.

La simplificación al considerar que toda la actividad sísmica se genera en tres fuentes sismogénicas volumétricas, es consistente con el nivel de detalle disponible en la caracterización de la actividad sísmica de la región. En efecto, en el contexto de un análisis probabilístico tradicional, cada falla activa conocida se hubiera considerado como una fuente sismogénica independiente con el eventual agregado de una fuente de sismicidad aleatoria o flotante.
Sin embargo, está claro que para el caso estudiado se carece de información de cada falla individual, suficiente para establecer una ley de Gutemberg-Richter para cada una de ellas. Sí en cambio, se dispone de suficiente información, tectónica y sismológica, para caracterizar las zonas definidas en el apartado anterior y postular una ley G-R regional. Con este tratamiento, es posible también contrastar los resultados con los publicados por diversos autores que realizaron trabajos similares en el pasado (Castano, 1987; INPRES, 1982; INPRES, 1993), en donde se comprueba que los resultados obtenidos para la Precordillera y la Zona de Benioff son razonables.

Los resultados del análisis probabilístico se representaron en primer lugar, como curvas de amenaza de pseudoaceleración con $5 \%$ de amortiguamiento, para periodos de $0.01 \mathrm{seg}, 0.2 \mathrm{seg}$ y $1 \mathrm{seg}$. Estas curvas se muestran en la Figura 7. La primera de ellas se puede asimilar a la de aceleración máxima del terreno.

En cada una se indica la contribución a la amenaza de las tres fuentes consideradas. Se calcularon también, los espectros de respuesta de pseudoaceleración para el 5\% de amortiguamiento para diferentes valores constantes del periodo de retorno, también denominados espectros de amenaza constante (Figura 8). Estos espectros no se pueden asociar a ningún evento real, ya que resultan de calcular aisladamente el valor de pseudoaceleración para cada periodo de vibración, con un valor constante especificado de periodo de retorno.

\section{Discusión}

La finalidad básica del estudio presentado en este trabajo es la definición de las solicitaciones sísmicas que deben utilizarse para el diseño y verificación de las obras que integran el proyecto en cuestión, para cada uno de los niveles de verificación a aplicar (Tabla 2). Para llegar a definir los movimientos sísmicos de verificación, es necesario utilizar una importante dosis de juicio crítico, con el fin de poder salvar los vacíos de información y las inconsistencias propias de las metodologías de análisis. El análisis crítico se discute en este apartado.

El análisis determinístico de la amenaza provee una serie de escenarios de verificación, que se asumen como la peor situación (en términos de magnitud y distancia) que puede generar cada una de las fuentes sismogénicas consideradas en el sitio de emplazamiento del proyecto. En base a este concepto, es relativamente sencillo seleccionar cuál es el evento crítico para cada tipo de estructura a considerar. Para estructuras de periodo corto dominan los terremotos cercanos, de baja magnitud, como son los generados por las fallas del Carrizal o Villa Corral. Para estructuras de periodo largo el evento que controla la amenaza es el correspondiente a la falla El Tigre, fundamentalmente por la posibilidad de generar efectos de directividad. Para obras geotécnicas, que tienen una importante capacidad de deformación 


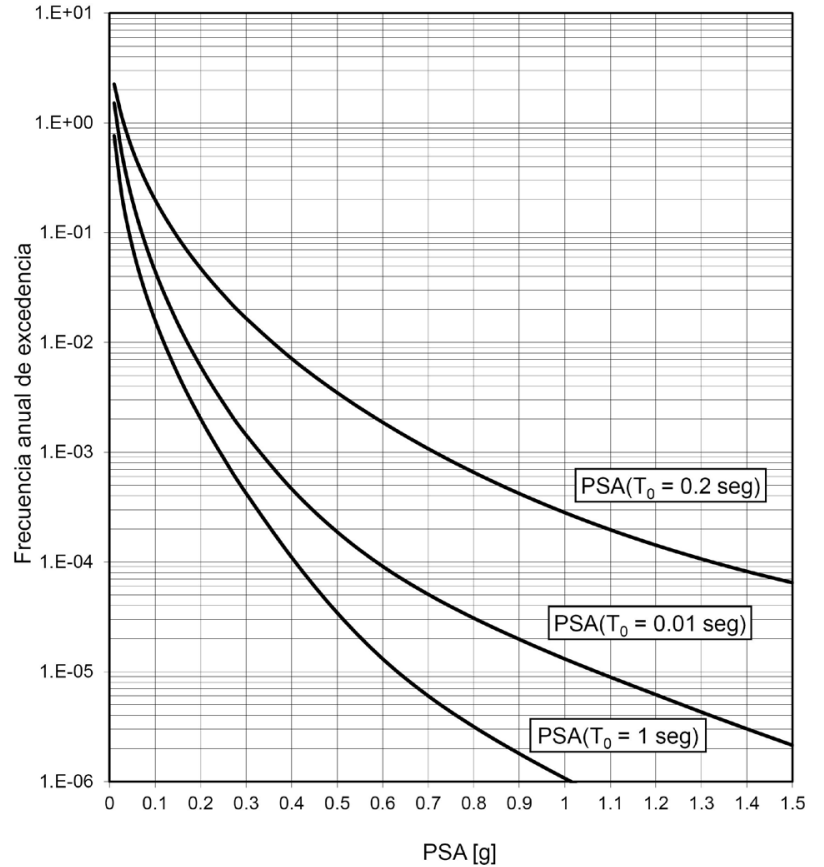

Figura 7. Curvas de amenaza de pseudoaceleración ( $5 \%$ de amortiguamiento) para el sitio de emplazamiento del Proyecto Casposo. La curva de amenaza para $\mathrm{T}_{0}=0.01 \mathrm{~s}$ se puede asumir como la correspondiente a la de aceleración máxima del terreno. La escala es logarítmica en el eje de las ordenadas y lineal en el eje de las abscisas.

permanente, es también importante considerar la duración del movimiento, que aumenta considerablemente con la magnitud. Por tanto, para el diseño de estas obras el evento que controla la seguridad será también el generado por la falla El Tigre, que es el de mayor magnitud.

Sin embargo, la selección del movimiento sísmico de verificación sobre la sola base del análisis determinístico requiere tomar la decisión del número de desviaciones estándar a considerar por sobre la media que predicen las relaciones de atenuación. Estas relaciones se formulan a partir de un análisis estadístico de los movimientos registrados y por lo tanto las predicciones que se hacen con ellas constan de un valor medio y una medida de su varianza. Abrahamson (2006) indica que, en la mayoría de las relaciones de atenuación, valores del parámetro de movimiento (aceleración máxima del terreno por ejemplo) de la media, más 2 ó 3 desviaciones estándar son físicamente posibles. Por tanto, el criterio de buscar la peor situación que puede presentarse para una determinada obra puede, a pesar de ser un concepto atractivo por su simplicidad, resultar de difícil aplicación en la práctica. En efecto, asumir un escenario con la máxima magnitud que puede desarrollar la falla a la menor distancia posible entre el sitio y la fuente, para luego adoptar el mayor movimiento posible que resulta de la relación de atenuación (digamos la media más tres desviaciones estándar) parece a todas luces un criterio excesivamente conservador. El problema se agrava aún más si se tiene en cuenta la variabilidad

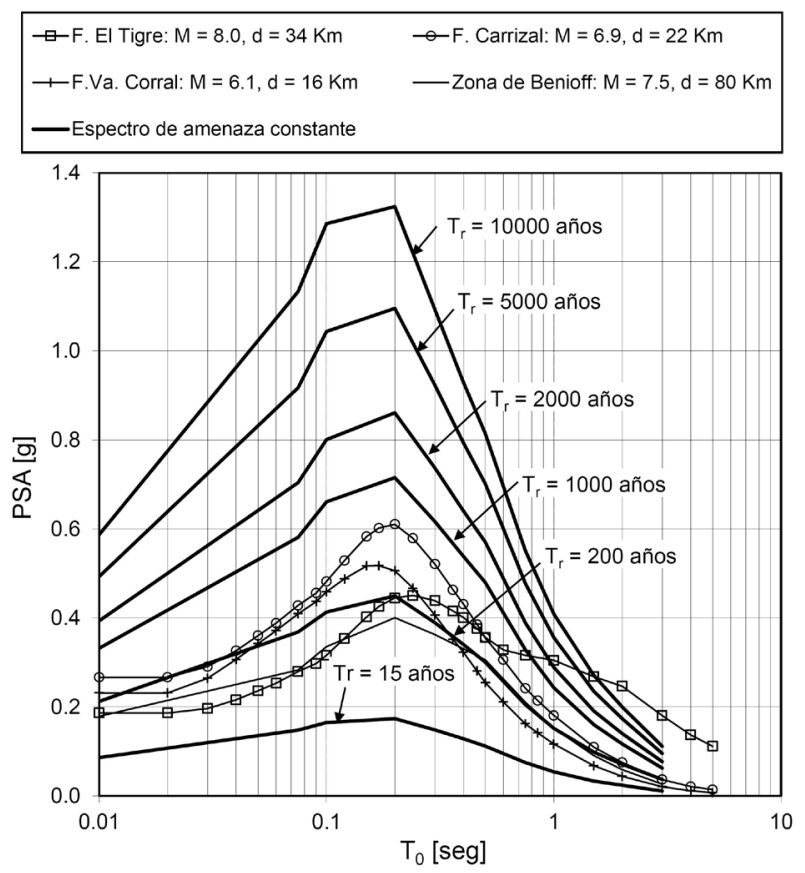

Figura 8. Espectros de amenaza constante obtenidos mediante análisis probabilístico de la amenaza, comparados con los espectros medios obtenidos del análisis determinístico de la amenaza. La escala es lineal en el eje de las ordenadas y logarítmica en el eje de las abscisas.

en las correlaciones que se han utilizado para determinar la magnitud potencial máxima asociada a cada una de las fallas consideradas. Como alternativa, la práctica de ingeniería habitual, al aplicar la metodología de análisis determinístico, es asumir que el máximo parámetro de movimiento corresponde a la media o a la media más una desviación estándar que resulta de la relación de atenuación. Esto se entiende como una práctica "razonablemente conservadora", pero evidentemente, no deja de ser una arbitrariedad (Abrahamson, 2000; Musson et al., 2005).

Por otro lado, el análisis probabilístico integra la contribución de todos los eventos considerados físicamente posibles o significativos en la modelación (es decir un rango de magnitudes y un rango de distancias a la ruptura) y también la variabilidad del movimiento que predicen las relaciones de atenuación adoptadas. El resultado es la frecuencia media anual de excedencia de cada valor de un cierto parámetro de movimiento (aceleración máxima del terreno por ejemplo) en el sitio analizado, lo que se conoce como la curva de amenaza. Con esto se obtiene una medida clara de la probabilidad de que la intensidad del movimiento en el sitio estudiado supere un cierto valor, en la vida útil de la obra. Esto facilita enormemente la decisión de cuál debe ser el nivel de intensidad de movimiento a utilizar en el diseño. Sin embargo la elección del evento (definido por la fuente sismogénica, la magnitud y la distancia al sitio) que origina ese movimiento de diseño ya no es tan evidente como resulta de un análisis determinístico. La selección 
de un evento es necesaria para poder definir el resto de las características del movimiento (duración, contenido de frecuencias, efectos de área epicentral) y poder así postular un acelerograma de diseño. Para poder seleccionar un evento en concreto se recurre al procedimiento de desagregación de la amenaza (Abrahamson, 2000; 2006) para investigar la contribución relativa de cada uno de esos eventos en la amenaza calculada. La Figura 9, muestra el resultado de la desagregación de la amenaza del movimiento de diseño con periodo de retorno de 1000 años, para la aceleración máxima del terreno y la pseudoaceleración correspondiente a periodos propios de 0.2 y 1 segundo. Esto permite, por ejemplo, buscar el evento más frecuente (la moda estadística) para adoptarlo como evento de diseño. También se puede utilizar la media estadística en lugar de la moda, aunque el primer criterio es más habitual en la actualidad (Abrahamson, 2006).

A pesar de que la metodología de análisis probabilístico está actualmente bien desarrollada, existe una dificultad intrínseca al método, cuando éste se intenta aplicar a la definición de la amenaza para la verificación de un conjunto de estructuras de diferente tipo y naturaleza, tal como ocurre en el caso estudiado. Eso se debe a que el comportamiento de cada estructura, y en definitiva también su falla, puede estar controlado por diferentes parámetros de movimiento, según el tipo de estructura de que se trate. Esto significa que la curva de amenaza obtenida, por ejemplo, para la aceleración máxima del terreno, puede ser determinante para un cierto tipo de estructura y completamente irrelevante para otro. El problema se complica aún más si consideramos que el parámetro de movimiento que controla el comportamiento estructural puede ser diferente, dependiendo del comportamiento deseado de la estructura (por ejemplo una estructura diseñada para comportarse en rango elástico o, por el contrario, para tener deformaciones permanentes) y que además hay problemas que están controlados por más de un parámetro de movimiento (por ejemplo la deformación permanente de un talud depende simultáneamente de la intensidad del movimiento, de la duración del mismo y del contenido de frecuencias). La solución a este inconveniente suele ser repetir el cálculo probabilístico para un conjunto de múltiples parámetros de movimiento, que se estiman relevantes. Por ejemplo, en el caso estudiado se analizaron, además de la aceleración máxima del terreno, un conjunto de valores de pseudoaceleración que corresponden a diferentes periodos propios, que se supone cubren el rango de periodos de las estructuras a verificar. El inconveniente de esta práctica es que al final del proceso de desagregación, en lugar de obtener un único escenario de verificación (el correspondiente al evento más frecuente por ejemplo), se obtienen múltiples escenarios, entre los cuales es difícil seleccionar uno en particular, puesto que en realidad se carece de un criterio objetivo para hacerlo. La selección final de escenarios de verificación se dificulta aún más si se reconoce que no todos los parámetros de movimiento

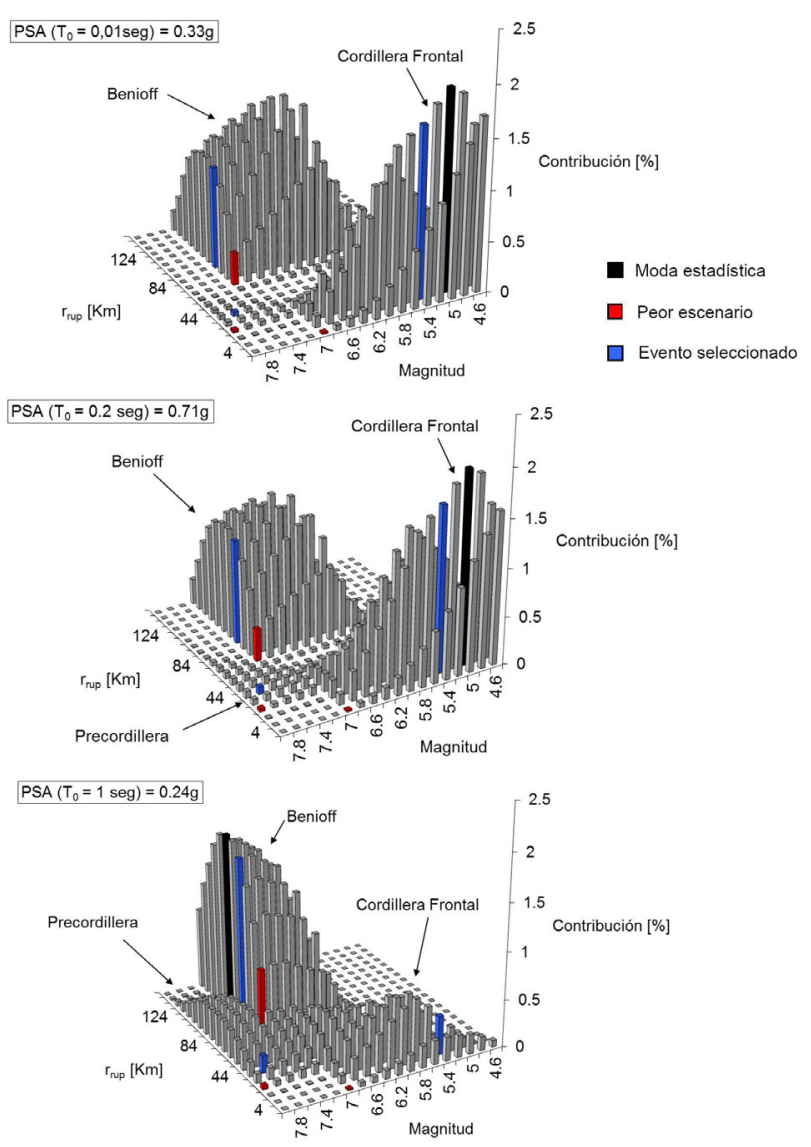

Figura 9. Desagregación de la amenaza para el $\mathrm{TMD}_{1000}$. Eventos seleccionados.

relevantes se han incluido en el análisis (en nuestro caso, por ejemplo, no se han analizado la duración del movimiento o los efectos de área epicentral). En realidad, sin un análisis estructural detallado de las obras a construir, es difícil saber a priori cuál es el conjunto de parámetros ideal a incluir en el análisis probabilístico. Es interesante notar aquí que este problema no se presenta en un análisis determinístico y en esto reside su fundamental ventaja. Se puede asegurar que para la gran mayoría, sino todos, de los problemas de ingeniería la capacidad destructiva de un movimiento sísmico aumenta con la magnitud del terremoto y disminuye con la distancia a la fuente. Por lo tanto, al considerar el peor escenario posible (máxima magnitud y mínima distancia), se tiene la seguridad de que el movimiento que resulta es el peor que puede generar cada una de las fuentes consideradas, independientemente del tipo de estructura a verificar.

La solución habitual al problema señalado para los análisis probabilísticos, es definir múltiples escenarios de verificación. De cada uno de esos escenarios se obtiene un movimiento sísmico diferente y por lo tanto en lugar de un único movimiento de verificación se tiene finalmente un conjunto de movimientos. Se espera entonces que el proyectista aplique todos esos movimientos a la 
verificación de la estructura y de esa manera garantizar que la estructura, en su diseño, ha sido sometida a toda la gama de movimientos que se presume que pueden ocurrir en el sitio de emplazamiento. Lógicamente la cantidad de movimientos de verificación no puede ser demasiado elevada pues esto complicaría en exceso el proceso de verificación.

La solución adoptada en este trabajo ha seguido la filosofía de definir múltiples escenarios y múltiples movimientos. Sin embargo, se han utilizado criterios adicionales para elegir dichos movimientos. Estos criterios se presentan a continuación y con la finalidad de discutir su utilidad.

En primer lugar se ha preferido incluir, para cada nivel de verificación, un sismo de más de una fuente sismogénica, aun cuando los resultados de la desagregación de la amenaza indiquen que se trata de eventos poco frecuentes. Esto se realiza para tener en cuenta los diferentes tipos de movimiento que generan cada uno de los estilos tectónicos presentes en el área estudiada.

Además, se han utilizado diferentes criterios de selección de movimientos, para los diferentes niveles de verificación (Tabla 4). Para el terremoto de operación normal (TON) se seleccionaron dos eventos que constituyen la moda estadística de las fuentes Benioff (la más frecuente) y Cordillera Frontal. Para el terremoto máximo de diseño con periodo de retorno de 1000 años $\left(\mathrm{TMD}_{1000}\right)$, en lugar de utilizar la moda o la media estadística para definir los escenarios de verificación, se eligieron eventos intermedios entre los eventos modales de cada fuente y los peores escenarios que resultan del análisis determinístico.

Los eventos modales, los peores y los seleccionados para el $\mathrm{TMD}_{1000}$ se señalan en la Figura 9. Finalmente, para el $\mathrm{TMD}_{10000}$ se utilizaron los eventos peores tal como se obtuvieron del análisis determinístico. La intensidad de los movimientos que se obtuvieron para cada escenario (definida por el espectro de respuesta de pseudoaceleraciones) se escaló para ajustarla al espectro de amenaza correspondiente (para $\operatorname{Tr}=15,1000$ ó 10000 años) en un rango de periodos propios adecuado. Los terremotos distantes y de gran magnitud (generados en la Precordillera y en la zona de Benioff) se ajustaron para el rango de periodos largos, en tanto que los terremotos cercanos de baja magnitud (de la Cordillera Frontal) se ajustaron para el rango de periodos cortos. La Figura 10 muestra los espectros ajustados. El escalado se hizo variando el número de desviaciones estándar que se suma al valor medio que predice la relación de atenuación correspondiente.

Los criterios y la metodología expuestos intentan salvar las dificultades propias de los procedimientos de análisis aplicados, obteniendo un resultado práctico aplicando criterios de ingeniería razonables. Así, parece razonable utilizar la moda para el caso del terremoto de operación normal, puesto que la verificación con este sismo tiene un objetivo puramente económico, como es asegurar una probabilidad predefinida para una detención de la operación del proyecto a consecuencia de un sismo. Por el contrario para los niveles de verificación más elevados $\left(\mathrm{TMD}_{1000} \mathrm{y}\right.$ $\mathrm{TMD}_{10000}$ ), en los que las consecuencias de una falla son progresivamente más graves y, además, para los que se admiten mayores daños en las obras, es razonable utilizar eventos gradualmente más próximos a los peores escenarios que surgirían de un análisis determinístico. Hacerlo así permite asegurar que se tomen en cuenta todos los efectos del movimiento que pueden presentarse en el sitio y que no pueden ser tenidos en cuenta en un análisis probabilístico de la amenaza. Por otro lado, la utilización del análisis probabilístico permite definir con objetividad la intensidad de los movimientos en el sitio para una probabilidad de excedencia prefijada, escalando los espectros de respuesta al nivel adecuado que surge de la curva de amenaza.

Tabla 4. Escenarios de verificación.

\begin{tabular}{|c|c|c|c|c|c|c|c|c|}
\hline Nivel & $\begin{array}{c}\operatorname{Tr} \\
\text { [años] }\end{array}$ & Desig. & $\mathrm{M}_{\mathrm{w}}$ & $\begin{array}{c}\mathrm{r}_{\text {rup }} \\
{[\mathrm{Km}]}\end{array}$ & $\begin{array}{c}\mathrm{H} \\
{[\mathrm{Km}]}\end{array}$ & $\varepsilon$ & $\begin{array}{c}\text { PGA } \\
{[\mathrm{g}]}\end{array}$ & Fuente/Mecanismo \\
\hline \multirow{2}{*}{ TON } & \multirow{2}{*}{15} & $15 / 1$ & 6 & 100 & 70 & 1 & 0.09 & Benioff / Intraplaca \\
\hline & & $15 / 2$ & 5 & 28 & --- & 1 & 0.09 & C. Frontal / F. Invers/Bloque ascend. \\
\hline \multirow{3}{*}{ TMD 1000} & \multirow{3}{*}{1000} & $1000 / 1$ & 5.4 & 12 & --- & 0.9 & 0.34 & C. Frontal / F. Invers / Bloque ascend. \\
\hline & & $1000 / 2$ & 7.4 & 100 & 100 & 1 & 0.3 & Benioff / Intraplaca \\
\hline & & $1000 / 3$ & 7.8 & 44 & --- & 0.5 & 0.17 & Precord. / F. Rumbo \\
\hline \multirow{4}{*}{$\begin{array}{c}\text { TMD } \\
10000\end{array}$} & \multirow{4}{*}{10000} & $10000 / 1$ & 5.8 & 12 & --- & 1.1 & 0.6 & C. Frontal / F. Invers / Bloque ascend. \\
\hline & & $10000 / 2$ & 6.8 & 20 & --- & 1.4 & 0.59 & C. Frontal / F. Invers / Bloque ascend. \\
\hline & & $10000 / 3$ & 7.4 & 76 & 70 & 1.6 & 0.59 & Benioff / Intraplaca \\
\hline & & $10000 / 4$ & 8 & 34 & --- & 1 & 0.29 & Precord. / F. Rumbo \\
\hline
\end{tabular}




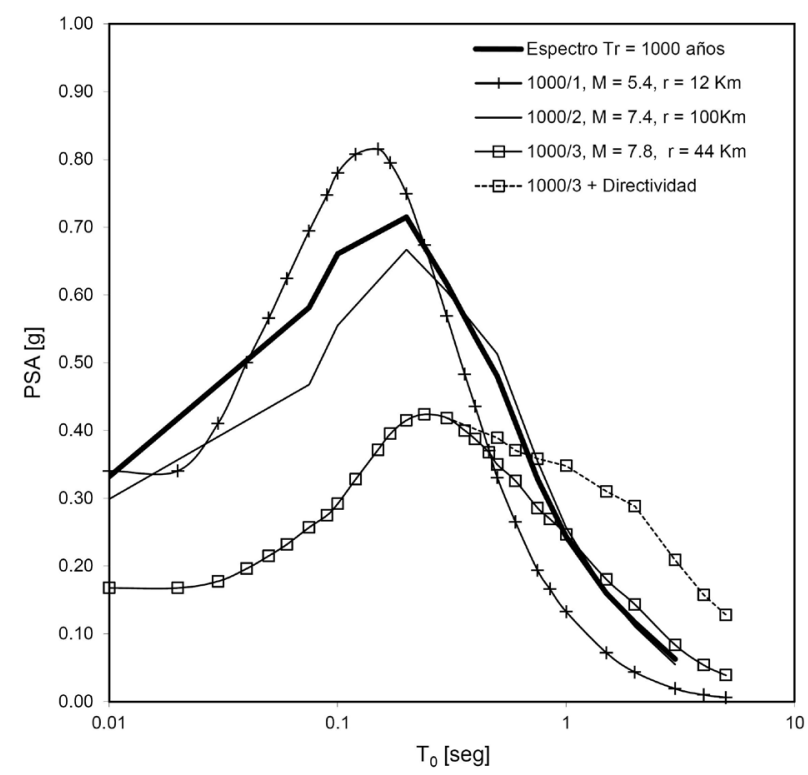

Figura 10. Espectros de respuesta de los movimientos de verificación seleccionados para el $\mathrm{TMD}_{1000}$, escalados para lograr el ajuste con el espectro de amenaza constante. La escala es lineal en el eje de las ordenadas y logarítmica en el eje de las abscisas.

\section{Conclusiones}

El presente trabajo presenta el caso de un análisis de amenaza sísmica para un proyecto minero ubicado en una zona sísmicamente activa. Las particularidades del sitio y de las obras proyectadas se tuvieron en cuenta al desarrollar la metodología de análisis aplicada. El artículo presenta todas las etapas que integran la evaluación donde fue necesaria la intervención de una serie de especialidades de la Geología y la Ingeniería Civil.

La estructura del margen precordillerano occidental es variada ya que, al norte del río San Juan se expresa en fallas con componente de desplazamiento de rumbo dextral y normal. Al sur de este río predominan los corrimientos y retrocorrimientos longitudinales combinados con fallas oblicuas de orientación Noroeste asociados a una notoria reactivación cuaternaria del relieve. Los datos disponibles acerca de edades de los depósitos y fallas cuaternarias son sumamente escasos, excepto para la falla El Tigre, así como resulta insuficiente el conocimiento sobre la geometría, cinemática y paleosismología de las principales fallas activas ubicadas en el flanco suroccidental de la Precordillera.

El área de proyecto no se considera expuesta directamente a severos problemas de remoción en masa inducidos por sismicidad, excepto en las áreas de mayor pendiente. Tampoco los fenómenos asociados a licuefacción constituyen un factor de riesgo significativo, por encontrarse ubicada el área sobre material grueso, tal como arenas y gravas. Por lo tanto, es probable que los mayores daños, en caso de ocurrencia de un sismo de elevada magnitud, sean directamente producidos por la sacudida sísmica, más que por procesos secundarios.

La metodología aplicada para el análisis de la amenaza intenta integrar toda la información disponible y obtener un resultado de aplicación práctica a un proyecto de ingeniería con particularidades propias. Condicionan la metodología, por un lado la información de partida incompleta y escasa, situación habitual en Sudamérica, y por otro lado la variedad de tipologías estructurales y obras involucradas en el proyecto y la posible ausencia de obras críticas, que justificarían el sólo uso de un análisis determinístico. La metodología utiliza dos procedimientos de análisis disponibles en la actualidad: probabilístico y determinístico, intentando aprovechar las ventajas de ambos y de integrar los resultados en un conjunto de movimientos de diseño que describan adecuadamente la amenaza sísmica en el sitio estudiado y para el conjunto de obras a construir.

\section{Agradecimientos}

Los autores expresan su agradecimiento a la empresa TROY RESOURCES ARGENTINA y al Ing. Eduardo Machuca por autorizar la presentación de este trabajo. Finalmente, se agradece a los evaluadores, Dres. Hans Diederix y Lluis Pujades por la revisión de este trabajo.

\section{Referencias}

Abrahamson, N.A., 2000, State of the practice of seismic hazard evaluation, Proceedings of Geological Engineering: Melbourne, Australia, Ervin MC, 659-685.

Abrahamson, N.A., 2006, Seismic hazard assessment: problems with current practice and future developments: First European Conference on Earthquake Engineering and Seismology, Geneva, Suiza, 1-17.

Abrahamson, N.A., Silva, W.J., 1997, Empirical response spectral attenuation relations for shalow crustal earthquakes: Seismological Research Letters, 68, 94-127.

Alvarado, P., Beck, S., 2006, Source characterization of the San Juan (Argentina). Crustal earthquakes of 15 January 1944 (Mw 7.0) and 11 June 1952 (Mw 6.8): Earth and Planetary Science Letters, 243, 615-631.

Barazangui, M., Isacks, B., 1976, Spatial distribution of earthquakes and subduction of the Nazca Plate beneath South America: Geology, 4, 686-692.

Bastías, H., 1986, Fallamiento cuaternario en la región sismotectónica de Precordillera: San Juan, Argentina, Facultad de Ciencias Exactas, Físicas y Naturales, Universidad Nacional de San Juan, Tesis Doctoral, 153p.

Bastías, H., Uliarte, E., Paredes, J., Sanchez, A., Bastías, J., Ruzycki, L., Perucca L., 1990, Neotectónica de la provincia de San Juan, en $11^{\circ}$ Congreso Geológico Argentino, Relatorio de Geología y Recursos Naturales de la Provincia de San Juan: San Juan, Argentina, Bordonaro, O. (Ed.), 228-245.

Castano, C., 1987, Actualización del estudio de riesgo sísmico y determinación de los parámetros para el diseño sismorresistente en el sitio de emplazamiento de la Presa El Tambolar: San Juan, Argentina, Gobierno de la Provincia de San Juan. Ministerio de Obras y Servicios Públicos. Secretaría de Recursos Hídricos, Informe Técnico, 223 p. 
Cortés, J., 1998, Tectónica de desplazamiento de rumbo en el borde sur de la depresión de Yalguaraz, Mendoza, Argentina: Revista de la Asociación Geológica Argentina, 53, 147-157.

Cortés, J.M., Cegarra, M., 2004, Plegamiento cuaternario transpresivo en el piedemonte suroccidental de la Precordillera sanjuanina: Revista de la Asociación Geológica Argentina, D7, 68-75.

Cortés, J., Costa, C.H., 1993, La deformación Cuaternaria pedemontana al norte de la Pampa Yalguaraz, margen occidental de la Precordillera de San Juan y Mendoza, en $12^{\circ}$ Congreso Geológico Argentino y $2^{\circ}$ Congreso de Exploración de Hidrocarburos: Buenos Aires, Argentina, Ramos, V. (Ed.), 241-245.

Cortés, J., Vinciguerra, P., Yamín, M.; Pasini, M., 1999, Tectónica Cuaternaria en la región andina del nuevo Cuyo, en Caminos, $\mathrm{R}$. (Ed.), Geología Argentina Anales 29: Buenos Aires, Argentina, Servicio Geológico Minero Argentino, 760-778.

Costa, C., Vita Finzi, C., 1996, Late Holocene intraplate faulting in the southeast Sierras Pampeanas, Argentina: Geology, 24, 1127-1130.

Costa, C., Machette, M., Dart, R., Bastías, H., Paredes, J., Perucca, L., Tello, G., Haller, K., 2000, Map and Database of Quaternary Faults and Folds in Argentina: Denver, United States Geological Survey, 75 p.

Costa, C., Murillo, V., Sagripanti, G., Gardini, C., 2001, Quaternary intraplate deformation in the southeastern Sierras Pampeanas, Argentina: Journal of Seismology, 5, 399-409.

Costa, C., Audemard, F., Bezerra, F., Lavenu, A., Machette, M., Paris, G., 2006, An overview of the main Quaternary deformation of South America: Revista de la Asociación Geológica Argentina, 61, 461-479.

Federal Emergency Management Agency (FEMA), 2000, Fema 356. Prestandard and commentary for the seismic rehabilitation of buildings: Washington, D.C, E.U.A., FEMA, 518p.

International Commission on Large Dams (ICOLD), 1989, Bulletin 72, Selecting seismic parameters for large dams: París, Francia, International Commission on Large Dams, 70p.

Instituto Nacional de Prevención Sísmica, (INPRES), 1982, Microzonificación sísmica del valle del Tulum: Provincia de San Juan. Resumen Ejecutivo: San Juan, Argentina, INPRES, 120 p.

Instituto Nacional de Prevención Sísmica, (INPRES), 1993, La verdadera dimensión del problema sísmico en la provincia de San Juan. Publicación Técnica $N^{\circ}$ 18: San Juan, Argentina, INPRES, 46 p.

Jordan, T., Gardeweg, M., 1989, Tectonic evolution of the late Cenozoic Central Andes, en Ben-Avraham, Z. (Ed.), Mesozoic and Cenozoic Evolution of the Pacific Margins: New York, U.S.A., Oxford University Press, 193-207.

Jordan, T., Isacks, B., Allmendinguer, R., Brewer, J., Ramos, V., Ando, C., 1983, Andean tectonics related to geometry of subducted Nazca plate: Geological Society of America Bulletin, 94, 341-361.

Kay, S., Mpodozis, C., Ramos, V., Munizaga, F., 1991, Magma source variations for mid-late Tertiary magmatic rocks associated with a shallowing subduction zone and a thickening crust in the central Andes $\left(28^{\circ}\right.$ to $\left.33^{\circ} \mathrm{S}\right)$, en Harmon, R.S., Rapela, C.W. (Eds.), Andean Magmatism and its Tectonic Setting: Boulder, Colorado, E.U.A., Geological Society of America, 113-137.

Mingorance, F., 1998, Evidencias de Paleoterremotos en la falla activa La Cantera-Cinturón de empuje de la Precordillera. San Juan, Argentina, en $10^{\circ}$ Congreso Latinoamericano de Geología y $6^{\circ}$ Congreso Nacional de Geología Económica, Actas 2: Buenos Aires, Argentina, Vujovich, G. (Ed.), 161-166.
Musson, R.M., Toro, G.R., Coppersmith, K.J., Bommer, J.J., Deichmann, N., Bungum, H., Cotton, F., Scherbaum, F., Slejko, D., Abrahamson, N.A., 2005, Evaluating hazard results for Switzerland and how not to do it: A discussion of Problems in the application of the SSHAC probability method for assessing earthquake hazards at Swiss nuclear power plants by J.U. Klügel: Engineering Geology, 82, 43-55.

Paredes, J., 1993, Geomorfología tectónica del borde occidental de la sierra del Tontal y su relación con la evaluación preliminar del peligro sísmico, en $12^{\circ}$ Congreso Geológico Argentino y $2^{\circ}$ Congreso de Hidrocarburos, Actas 6: Mendoza, Argentina, Ramos, V. (Ed.), 333-337.

Quartino, B., Zardini, R., Amos, A., 1971, Estudio y exploración geológica de la región Barreal-Calingasta, provincia de San Juan, República Argentina: Buenos Aires, Argentina: Asociación Geológica Argentina, $184 \mathrm{p}$.

Ramos, V., 1988, The tectonics of the Central Andes, $30^{\circ}-33^{\circ}$ latitude, en Clark, S., Burchfield, D., Suppe, J. (Eds.), Processes in Continental Litospheric Deformation: Boulder, E.U.A., Geological Society of America, 31-54.

Siame, L., Bourles, D., Sebrier, M., Bellier, O., Castano, J., Araujo, M., Pérez, M., Raisbeck, G., Yiou, F., 1997, Cosmogenic dating from 20 to $700 \mathrm{ka}$ of a series of alluvial fan surfaces affected by El Tigre fault, Argentina: Geology, 25, 975-978.

Slemmons, D.B., 1982, Determination of design earthquake magnitudes for microzonation, in Proceedings of the Third International Earthquake Microzonation Conference Vol. 1: Washington, D.C., E.U.A., National Science Foundation, 119-130.

Smalley, R., Isacks, B., 1987, A high resolution local network study of the Nazca Plate Wadati-Benioff zone under western Argentina: Journal of Geophysical Research, 92, 13903-13912.

Smalley, R., Isacks, B., 1990, Seismotectonics of thin and thick-skinned deformation in the Andean foreland from local network data: Evidence for a seismogenic lower crust: Journal of Geophysical Research, 95, 12487-12498

Smalley R., Pujol, J., Regnier, M., Chiu, J.M., Chatelain, Jl., Isacks, B.L., Araujo, M., Puebla, N., 1993, Basement seismicity beneath the Andean Precordillera thin-skinned thrust belt and implications for crustal and lithospheric behavior: Tectonics, 12, 63-76.

Somerville, P.G., Smith, N.F., Graves, R.W., Abrahamson, N.A., 1997, Modification of empirical strong ground motion attenuation relations to include the amplitude and duration effects of rupture directivity: Seismological Research Letters, 68, 199-222.

Wells, D., Coppersmith, K., 1994, New empirical Relationships among Magnitude, Rupture length, Rupture width, Rupture area and Surface displacements: Bulletin of the Seismological Society of America, 84, 974-1002.

Youngs, R.R., Chiou, S.-J., Silva, W.J., Humphrey, J.R., 1997, Strong ground motion attenuation relationships for subduction zone earthquakes: Seismological Research Letters, 68, 58-73.

Manuscrito recibido: Noviembre 27, 2011.

Manuscrito corregido recibido: Enero 23, 2012.

Manuscrito aceptado: Enero 31, 2012. 